\title{
The Generalized Dahlquist Constant with Applications in Synchronization Analysis of Typical Neural Networks via General Intermittent Control
}

\author{
Zhang Qunli \\ Department of Mathematics, Heze University, Heze 274015, Shandong, China \\ Correspondence should be addressed to Zhang Qunli, qunli-zhang@126.com \\ Received 11 January 2011; Revised 8 May 2011; Accepted 7 June 2011 \\ Academic Editor: Tomasz G. Smolinski
}

Copyright (c) 2011 Zhang Qunli. This is an open access article distributed under the Creative Commons Attribution License, which permits unrestricted use, distribution, and reproduction in any medium, provided the original work is properly cited.

\begin{abstract}
A novel and effective approach to synchronization analysis of neural networks is investigated by using the nonlinear operator named the generalized Dahlquist constant and the general intermittent control. The proposed approach offers a design procedure for synchronization of a large class of neural networks. The numerical simulations whose theoretical results are applied to typical neural networks with and without delayed item demonstrate the effectiveness and feasibility of the proposed technique.
\end{abstract}

\section{Introduction}

Since its introduction by Pecora and Carrol in 1990, synchronization of chaotic systems [1-10] is of great practical significance and has received great interest in recent years. In the above literature, the approach applied to stability analysis is basically Lyapunov's method. As we all know, the construction of a proper Lyapunov function becomes usually very skillful, and Lyapunov's method does not specifically describe the convergence rate near the equilibrium of the system. Hence, there is little compatibility among all of the stability criteria obtained so far.

The concept named the generalized Dahlquist constant [11] has been applied to the investigation of impulsive synchronization $[12,13]$ analysis.

Intermittent control [14-18] has been used for a variety of purposes in engineering fields such as manufacturing, transportation, air-quality control, and communication. Synchronization using an intermittent control method has been discussed [15-18]. Compared with continuous control methods [2-10], intermittent control is more efficient when the system output is measured intermittently rather than continuously. Our interest focuses on the class of intermittent control with time duration, wherein the control is activated in certain nonzero time intervals and is off in other time intervals. A special case of such a control law is of the form

$$
U(t)= \begin{cases}-k(y(t)-x(t)), & (n T \leq t<n T+\delta), \\ 0, & (n T+\delta \leq t<(n+1) T),\end{cases}
$$

where $k$ denotes the control strength, $\delta>0$ denotes the switching width, and $T$ denotes the control period. In this paper, based on the generalized Dahlquist constant and the Gronwall inequality, a general intermittent controller

$$
U(t)= \begin{cases}-k(y(t)-x(t)), & (h(n) T \leq t<h(n) T+\delta), \\ 0, & (h(n) T+\delta \leq t<h(n+1) T),\end{cases}
$$

is designed, where $h(n)$ is a strictly monotone increasing function on $n$ with $h(0)=0$ then sufficient yet generic criteria for synchronization of typical neural networks with and without delayed item are obtained.

This paper is organized as follows. In Section 2, some necessary background materials are presented, and a simple configuration of coupled neural networks is formulated. Section 3 deals with synchronization. The theoretical results 
are applied to typical chaotic neural networks, and numerical simulations are shown in this section. Finally, some concluding remarks are given in Section 4.

\section{Formulations}

Let $X$ be a Banach space endowed with the Euclidean norm $\|\cdot\|$, that is, $\|x\|=\sqrt{x^{T} x}=\sqrt{\langle x, x\rangle}$, where $\langle\cdot, \cdot \cdot\rangle$ is inner product, and, let $\Omega$ be a open subset of $X$. We consider the following system:

$$
\frac{d x(t)}{d t}=F(x(t))+G(x(t-\tau))
$$

where $F, G$ are nonlinear operators defined on $\Omega, x(t), x(t-$ $\tau) \in \Omega, \tau$ is a time-delayed positive constant, and $F(0)=$ $0, G(0)=0$.

Definition 1. System (3) is called to be exponentially stable on a neighborhood $\Omega$ of the equilibrium point if there exist constants $\mu>0, M>0$, such that

$$
\|x(t)\| \leq M e^{-\mu t}\left\|x_{0}\right\|(t \geq 0)
$$

where $x(t)$ is any solution of (3) initiated from $x\left(t_{0}\right)=x_{0}$.

Definition 2 (see [11]). Suppose $\Omega$ is an open subset of Banach space $X$, and $F: \Omega \rightarrow X$ is an operator.

The constant

$$
\alpha(F)=\sup _{x, y \in \Omega, x \neq y} \frac{1}{\|x-y\|} \lim _{r \rightarrow+\infty} f(r)
$$

is called to be the generalized Dahlquist constant of $F$ on $\Omega$, where $f(r)=\|(F+r I) x-(F+r I) y\|-r\|x-y\|$; here, denote by $F+r I$ the operator mapping every point $x \in \Omega$ onto $F(x)+r x$.

For $r \geq 0$,

$$
\begin{aligned}
f(r) & =\|(F+r I) x-(F+r I) y\|-r\|x-y\| \\
& =\sqrt{(k(r))^{T} k(r)}-r \sqrt{(x-y)^{T}(x-y)} \\
& =f_{1}(r)-f_{2}(r)
\end{aligned}
$$

where $k(r)=F(x)-F(y)+r(x-y), f_{1}(r)=$ $\sqrt{(k(r))^{T} k(r)}, f_{2}(r)=r \sqrt{(x-y)^{T}(x-y)}$

$$
\begin{aligned}
\frac{d f_{1}(r)}{d r} & =\frac{(F(x)-F(y))^{T}(x-y)+r\|x-y\|^{2}}{f_{1}(r)} \\
& =\frac{f_{3}(r)}{f_{1}(r)}, \\
\frac{d f_{2}(r)}{d r} & =\sqrt{(x-y)^{T}(x-y)},
\end{aligned}
$$

where $f_{3}(r)=(F(x)-F(y))^{T}(x-y)+r\|x-y\|^{2}$. According to the Cauchy-Bunie Khodorkovsky inequality, we obtain

$$
\begin{aligned}
&\left(f_{1}(r) \sqrt{(x-y)^{T}(x-y)}\right)^{2}-\left(f_{3}(r)\right)^{2} \\
&=\|F(x)-F(y)\|^{2}\|x-y\|^{2}-\left((F(x)-F(y))^{T}(x-y)\right)^{2} \\
&=\langle F(x)-F(y), F(x)-F(y)\rangle\langle x-y, x-y\rangle \\
&-(\langle F(x)-F(y), x-y\rangle)^{2} \geq 0 .
\end{aligned}
$$

Therefore,

$$
\left|f_{1}(r) \sqrt{(x-y)^{T}(x-y)}\right| \geq\left|f_{3}(r)\right| \geq f_{3}(r) .
$$

That is

$$
\begin{gathered}
f_{1}(r) \sqrt{(x-y)^{T}(x-y)} \geq f_{3}(r), \\
\frac{d f_{1}(r)}{d r}-\frac{d f_{2}(r)}{d r} \leq 0, \\
\frac{d f(r)}{d r} \leq 0 .
\end{gathered}
$$

So the function $f(r), r \geq 0$, is monotone decreasing; thus, the limit

$$
\lim _{r \rightarrow+\infty} f(r)
$$

exists.

\section{Synchronization Analysis and Examples}

Theorem 3. If the operator $G$ in the system (3) satisfies

$$
\|G(x)-G(y)\| \leq l\|x-y\|
$$

for any $x, y \in \Omega$, where $l$ is a positive constant, then two solutions, $x(t)$ and $y(t)$, respectively, initiated from $x\left(t_{0}\right)=$ $x_{0} \in \Omega, y\left(t_{0}\right)=y_{0} \in \Omega$ satisfy

$$
\|x-y\| \leq\left\|x_{0}-y_{0}\right\| \exp \left\{\lambda\left(t-t_{0}\right)\right\}, \quad \forall t \geq 0,
$$

where $\lambda=\alpha(F)+\exp \{-\alpha(F) \tau\} l$.

Proof. Assume $x(t)$ and $y(t)$ are the solutions of (3), respectively, under the initial conditions $x\left(t_{0}\right)=x_{0} \in \Omega$, $y\left(t_{0}\right)=y_{0} \in \Omega$. We have

$$
\begin{aligned}
\left(e^{r t} x(t)\right)_{t}^{\prime} & =r e^{r t} x(t)+e^{r t} F(x(t))+e^{r t} G(x(t-\tau)) \\
& =e^{r t}(F+r I) x(t)+e^{r t} G(x(t-\tau))
\end{aligned}
$$

for all $t \geq 0$ and $r>0$.

For all $x_{0}, y_{0} \in \Omega, t>s \geq 0$,

$$
e^{r t}[x(t)-y(t)]=e^{r s}[x(s)-y(s)]+\int_{s}^{t} k(r, u) d u,
$$


where $k(r, u)=e^{r u}[(F+r I) x(u)-(F+r I) y(u)+(G(x(u-$ $\tau))-G(y(u-\tau)))]$.

So

$$
e^{r t}\|x(t)-y(t)\|-e^{r s}\|x(s)-y(s)\| \leq \int_{s}^{t} h(r, u) d u
$$

where $h(r, u)=e^{r u}(\|(F+r I) x(u)-(F+r I) y(u)\|+\| G(x(u-$ $\tau))-G(y(u-\tau)) \|)$.

Then for all $t \geq 0$, we infer that

$$
e^{r t}(\|x(t)-y(t)\|)_{t}^{\prime} \leq h(r, t) .
$$

Therefore, we obtain

$$
e^{r t}(\|x(t)-y(t)\|)_{t}^{\prime} \leq h(r, t)-r e^{r t}\|x(t)-y(t)\| .
$$

Letting $r \rightarrow+\infty$, then

$$
\begin{aligned}
(\|x(t)-y(t)\|)_{t}^{\prime} \leq & \alpha(F)\|x(t)-y(t)\| \\
& +\|G(x(t-\tau))-G(y(t-\tau))\| \\
\leq & \alpha(F)\|x(t)-y(t)\| \\
& +l\|x(t-\tau)-y(t-\tau)\| .
\end{aligned}
$$

Integrating inequality (19) over $\left[t_{0}, t\right]$, we have

$$
\begin{aligned}
\|x(t)-y(t)\| \leq & e^{\alpha(F)\left(t-t_{0}\right)}\left\|x_{0}-y_{0}\right\| \\
& \quad+\int_{t_{0}}^{t} e^{\alpha(F)(t-s)} l\|x(s-\tau)-y(s-\tau)\| d s .
\end{aligned}
$$

That is

$$
\begin{aligned}
& e^{-\alpha(F)\left(t-t_{0}\right)}\|x(t)-y(t)\| \leq\left\|x_{0}-y_{0}\right\| \\
& +\int_{t_{0}}^{t} e^{-\alpha(F)\left(s-t_{0}\right)} l\|x(s-\tau)-y(s-\tau)\| d s, \\
& \quad \leq\left\|x_{0}-y_{0}\right\| \\
& \quad+e^{-\alpha(F) \tau} l \int_{t_{0}-\tau}^{t-\tau} e^{-\alpha(F)\left(s-t_{0}\right)}\|x(s)-y(s)\| d s .
\end{aligned}
$$

Using the Gronwall inequality [19, 20], we have

$$
e^{-\alpha(F)\left(t-t_{0}\right)}\|x(t)-y(t)\| \leq\left\|x_{0}-y_{0}\right\| \exp \left\{e^{-\alpha(F) \tau} l\left(t-t_{0}\right)\right\} .
$$

Then

$$
\|x(t)-y(t)\| \leq\left\|x_{0}-y_{0}\right\| \exp \left\{\left(\alpha(F)+e^{-\alpha(F) \tau} l\right)\left(t-t_{0}\right)\right\} .
$$

Let system (3) be the drive system, and we consider the response system

$$
\frac{d y(t)}{d t}=F(y(t))+G(y(t-\tau))+U(t),
$$

where $x, y \in R^{n}$ are the state variables, $F(\cdot), G(\cdot)$ are nonlinear operators, $U(t)$ is a feedback control term, and

$$
U(t)= \begin{cases}-k(y(t)-x(t)), & (h(n) T \leq t<h(n) T+\delta) \\ 0, & (h(n) T+\delta \leq t<h(n+1) T)\end{cases}
$$

where $k$ denotes the control strength, $T$ is the control period, $\delta$ is called the control width, and $h(n)$ is a strictly monotone increasing function on $n$ with $h(0)=0$.

In this paper, our goal is to design suitable function, $h(n)$ and suitable parameters, $\delta, T$, and $k$ such that system (24) synchronizes to system (3).

Subtract (3) from (24), the error system is obtained

$$
\frac{d e(t)}{d t}=\left\{\begin{array}{l}
F(y(t))-F(x(t)) \\
+G(y(t-\tau))-G(x(t-\tau))-k e(t), \\
\quad(h(n) T \leq t<h(n) T+\delta), \\
F(y(t))-F(x(t)) \\
+G(y(t-\tau))-G(x(t-\tau)), \\
\quad(h(n) T+\delta \leq t<h(n+1) T),
\end{array}\right.
$$

where $e=y-x$. Then we have the following result.

Theorem 4. Suppose that the operator $G$ in the systems (3) and (24) satisfies condition (12), and $\alpha(F)$ is defined as in Definition 2, and $\lambda=\alpha(F)+\exp \{-\alpha(F) \tau\}$ l. Then the synchronization of (3) and (24), given in (26), is asymptotically stable if the parameters $\delta, T$, and $k$ are such that

$$
\inf \left((r+\lambda) \delta \frac{h^{-1}(t-\delta / T)}{t}-\lambda\right)>0
$$

where $r=k-\lambda>0, h^{-1}(\cdot)$ is inverse function of the function $h(\cdot)$.

Proof. From Theorem 3, we can get the conclusion as follows:

$$
\|e(t)\| \leq\|e(h(n) T)\| \exp \{-r(t-h(n) T)\}
$$

for any $h(n) T \leq t<h(n) T+\delta$,

$$
\|e(t)\| \leq\|e(h(n) T+\delta)\| \exp \{\lambda(t-h(n) T-\delta)\}
$$

for any $h(n) T+\delta \leq t<h(n+1) T$. 
Consider conditions (28) and (29), and we can get the conclusion that

$$
\begin{gathered}
\|e(t)\| \leq\left\{\begin{array}{c}
\|e(0)\| \exp \{-r t+(r+\lambda) h(n) T-n(r+\lambda) \delta\}, \\
(h(n) T \leq t<h(n) T+\delta), \\
\|e(0)\| \exp \{\lambda t-(n+1)(r+\lambda) \delta\} \\
(h(n) T+\delta \leq t<h(n+1) T),
\end{array}\right. \\
\leq\left\{\begin{array}{c}
\|e(0)\| \exp \left\{-\left((r+\lambda) \delta \frac{h^{-1}(t-\delta / T)}{t}-\lambda\right) t\right\}, \\
(h(n) n T \leq t<h(n) T+\delta), \\
\|e(0)\| \exp \left\{-\left((r+\lambda) \delta \frac{h^{-1}(t / T)}{t}-\lambda\right) t\right\}, \\
(h(n) T+\delta \leq t<h(n+1) T) .
\end{array}\right.
\end{gathered}
$$

When $t \rightarrow \infty,\|e(t)\| \rightarrow 0$ is obtained under condition (27) and (26) becomes asymptotically stable.

Corollary 5. Letting $G(x(t-\tau))=0, \lambda=\alpha(F)$ be defined as in Definition 2, and condition (27) is satisfied, then result similar to Theorem 4 is obtained.

Corollary 6. Supposing that $h(n)=p n, p>0$, the operator $G$ in the systems (3) and (24) satisfies condition (12), and $\alpha(F)$ is defined as in Definition 2, and $\lambda=\alpha(F)+\exp \{-\alpha(F) \tau\} l$ then; the synchronization of (3) and (24), given in (26), is asymptotically stable if the parameters $\delta, T$, and $k$ are such that

$$
(r+\lambda) \delta \frac{1}{p T}-\lambda>0
$$

where $r=k-\lambda>0$.

In the simulations of the following examples, we always choose $T=5, k=10$ and make use of the norm $\|x\|=$ $\sqrt{x^{T} x}$, where $x \in R^{n}$.

Example 7. Consider a typical delayed Hopfield neural network [21-23] with two neurons:

$$
\dot{x}(t)=-C x(t)+A f(x(t))+B f(x(t-\tau)),
$$

where $x(t)=\left(x_{1}(t), x_{2}(t)\right)^{T}, f(x(t))=\left(\tanh \left(x_{1}(t)\right)\right.$, $\left.\tanh \left(x_{2}(t)\right)\right)^{T}, \tau=(1)$, and $C=\left(\begin{array}{cc}1 & 0 \\ 0 & 1\end{array}\right), A=\left(\begin{array}{cc}2.0 & -0.1 \\ -5.0 & 3.0\end{array}\right)$, with $B=\left(\begin{array}{c}-1.5-0.1 \\ -0.2\end{array}-2.5\right)$.

It should be noted that the network is actually a chaotic delayed Hopfield neural network.

Equation (32) is considered as the drive system, and the response system is defined as follows:

$$
\begin{gathered}
\dot{y}(t)=-C y(t)+A f(y(t))+B f(y(t-\tau))+U(t), \\
y\left(t_{0}\right)=y_{0} .
\end{gathered}
$$

We calculate and get the value $l<9.15, \alpha(F) \leq 0.7993$, where $F(x(t))=-C x(t)+A f(x(t)), G(x(t-\tau))=B f(x(t-$ $\tau)$ ). Choose $h(n)=n, \delta=4$, and it is easy to verify that condition (31) is satisfied. Let the initial condition be

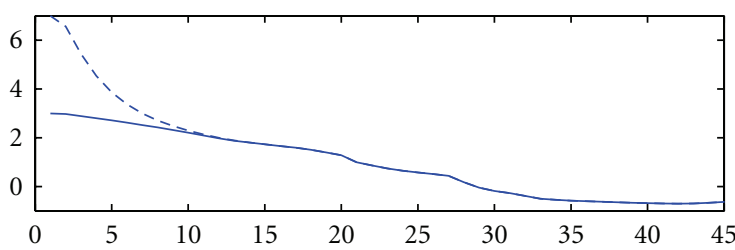

(a)

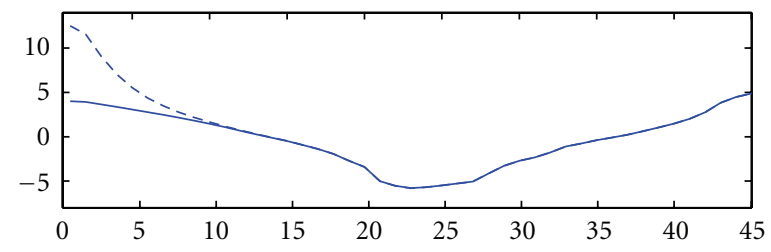

(b)

Figure 1: (a) Synchronization of $x_{1}(t)$ and $y_{1}(t)$. (b) Synchronization of $x_{2}(t)$ and $y_{2}(t)$.

$\left(\begin{array}{llll}x_{1} & x_{2} & y_{1} & y_{2}\end{array}\right)^{T}=\left(\begin{array}{llll}3 & 4 & 7 & 12.5\end{array}\right)^{T}$. Then it can be clearly seen in Figure 1 that the drive system (32) synchronizes with the response system (33).

Example 8. Considering a typical delayed chaotic neural network (29) with two neurons [24, 25] as the drive system, (31) as the response system, where $x(t)=\left(x_{1}(t), x_{2}(t)\right)^{T}$, $f(x(t))=\left(f_{1}\left(x_{1}(t)\right), f_{2}\left(x_{2}(t)\right)\right)^{T}, f_{i}\left(x_{i}(t)\right)=0.5\left(\left|x_{i}(t)+1\right|-\right.$ $\left.\left|x_{i}(t)-1\right|\right), i=1,2, \tau=(1), C=\left(\begin{array}{ll}1 & 0 \\ 0 & 1\end{array}\right), A=\left(\begin{array}{cc}1+\pi / 4 & 20 \\ 0.1 & 1+\pi / 4\end{array}\right)$, with $B=\left(\begin{array}{cc}-1.3 \sqrt{2} \pi / 4 & 0.1 \\ 0.1 & -1.3 \sqrt{2} \pi / 4\end{array}\right)$.

It is easily seen that the operator $f(x(t))$ is differential on $x$ in Example 7, but the operator $f(x(t))$ is not so in this example.

We calculate and get the value $l<1.3 \sqrt{2} \pi / 2+0.2, \alpha(F) \leq$ 1.0855, where $F(x(t))=-C x(t)+A f(x(t)), G(x(t-\tau))=$ $B f(x(t-\tau))$. Choose $h(n)=2 n, \delta=4$, and it is easy to verify that condition (31) is satisfied. Let the initial condition be $\left(\begin{array}{llll}x_{1} & x_{2} & y_{1} & y_{2}\end{array}\right)^{T}=\left(\begin{array}{lllll}3 & 4 & 17 & 12.8\end{array}\right)^{T}$. Then the synchronization property of this example can be clearly seen in Figure 2.

Example 9. Consider an autonomous Hopfield neural network with four neurons $[26,27]$ :

$$
\dot{x}(t)=-C x(t)+A f(x(t)),
$$

where $x(t)=\left(x_{1}(t), x_{2}(t), x_{3}(t), x_{4}(t)\right)^{T}, f(x(t))=\left(\tanh \left(x_{1}(t)\right)\right.$, $\left.\tanh \left(x_{2}(t)\right), \tanh \left(x_{3}(t)\right), \tanh \left(x_{4}(t)\right)\right)^{T}$, and

$$
C=\left(\begin{array}{llll}
1 & 0 & 0 & 0 \\
0 & 1 & 0 & 0 \\
0 & 0 & 1 & 0 \\
0 & 0 & 0 & 1
\end{array}\right),
$$

$$
A=\left(\begin{array}{cccc}
0.85 & -2 & -0.5 & 0.5 \\
1.8 & 1.15 & 0.6 & 0.3 \\
1.1 & 1.21 & 2.5 & 0.05 \\
0.1 & -0.4 & -1.5 & 1.45
\end{array}\right)
$$




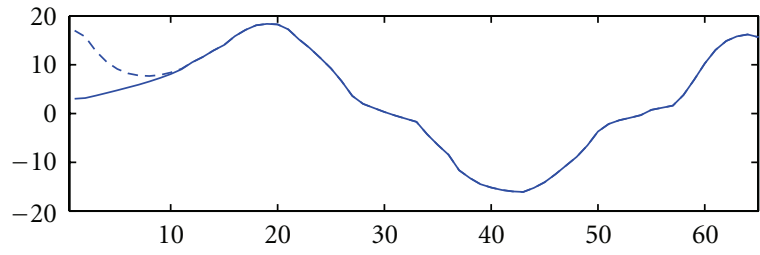

(a)

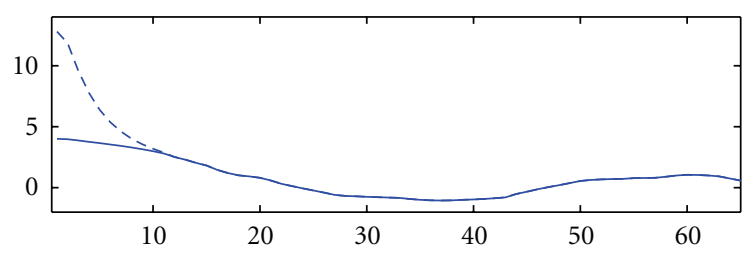

(b)

Figure 2: (a) Synchronization of $x_{1}(t)$ and $y_{1}(t)$. (b) Synchronization of $x_{2}(t)$ and $y_{2}(t)$.

Das II et al. [26] have reported that the system (34) posseses a chaotic behavior.

Equation (34) is considered as the drive system, and the response system is defined as follows:

$$
\begin{gathered}
\dot{y}(t)=-C y(t)+A f(y(t))+U(t), \\
y\left(t_{0}\right)=y_{0} .
\end{gathered}
$$

We calculate and get the value $\alpha(F) \leq 1.4369$, where $F(x(t))=-C x(t)+A f(x(t))$ and choose $h(n)=n / 2, \delta=$ 2. It is easy to verify that condition (31) is satisfied. Let the initial condition be $\left(\begin{array}{llllllll}x_{1} & x_{2} & x_{3} & x_{4} & y_{1} & y_{2} & y_{3} & y_{4}\end{array}\right)^{T}=$ $(2-1-21765.49)^{T}$. Then it can be clearly seen in Figure 3 that the drive system (34) synchronizes with the response system (36).

Example 10. Consider a typical hyperchaotic neural network (32) with two neurons [28] as the drive system, (33) as the response system, where $x(t)=\left(x_{1}(t), x_{2}(t), x_{3}(t), x_{4}(t)\right)^{T}$, $f(x(t))=\left(0,0,0,\left|x_{4}+1\right|-\left|x_{4}-1\right|\right)^{T}$, and

$$
\begin{gathered}
C=\left(\begin{array}{cccc}
0 & 0 & 1 & 1 \\
0 & -2 & -1 & 0 \\
-14 & 14 & 0 & 0 \\
-100 & 0 & 0 & 100
\end{array}\right), \\
A=\left(\begin{array}{lllc}
0 & 0 & 0 & 0 \\
0 & 0 & 0 & 0 \\
0 & 0 & 0 & 0 \\
0 & 0 & 0 & 100
\end{array}\right) .
\end{gathered}
$$

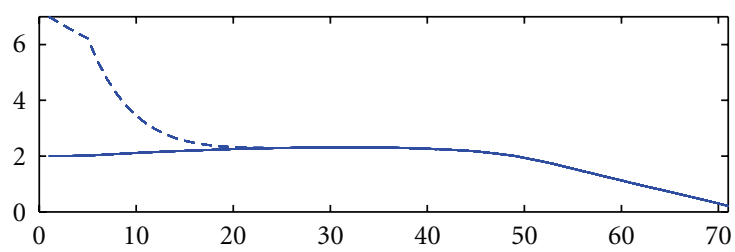

(a)

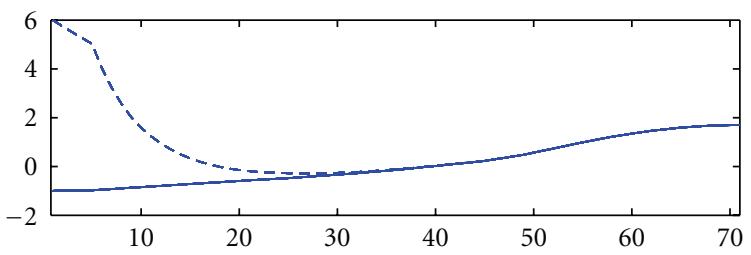

(b)

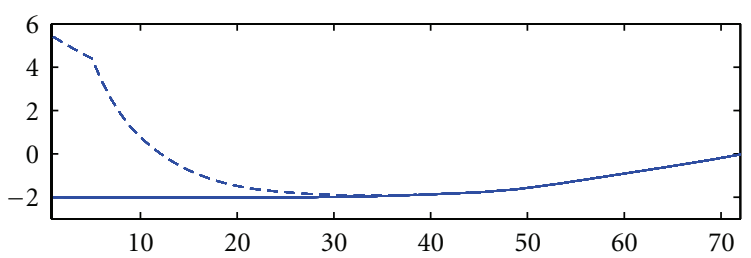

(c)

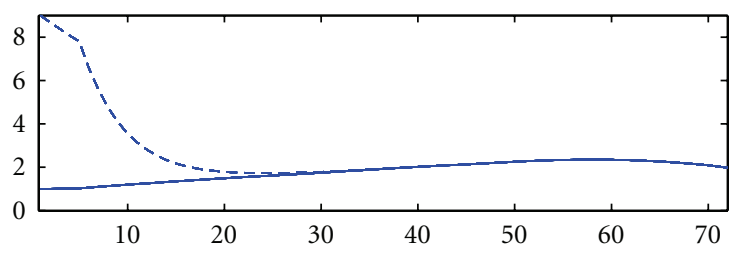

(d)

Figure 3: (a) Synchronization of $x_{1}(t)$ and $y_{1}(t)$. (b) Synchronization of $x_{2}(t)$ and $y_{2}(t)$. (c) Synchronization of $x_{3}(t)$ and $y_{3}(t)$. (d) Synchronization of $x_{4}(t)$ and $y_{4}(t)$.

We calculate and get the value $\alpha(F) \leq 14.8559$, where $F(x(t))=-C x(t)+A f(x(t))$ and choose $h(n)=n^{2} /$ $(n+1), \delta=2$. It is easy to verify that the condition (27) is satisfied. Letting the initial condition be

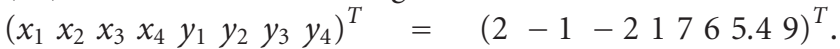
Then the synchronization property of this example can be clearly seen in Figure 4.

\section{Conclusion}

Approaches for synchronization of two coupled neural networks which use the nonlinear operator named the generalized Dahlquist constant and the general intermittent control have been presented in this paper. Strong properties of global and asymptotic synchronization have been achieved in a finite number of steps. The techniques have been successfully applied to typical neural networks. Numerical simulations have verified the effectiveness of the method. 


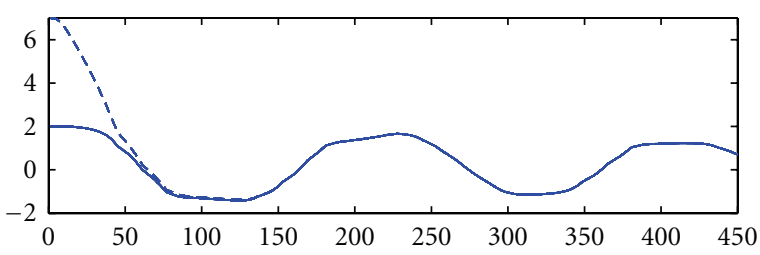

(a)

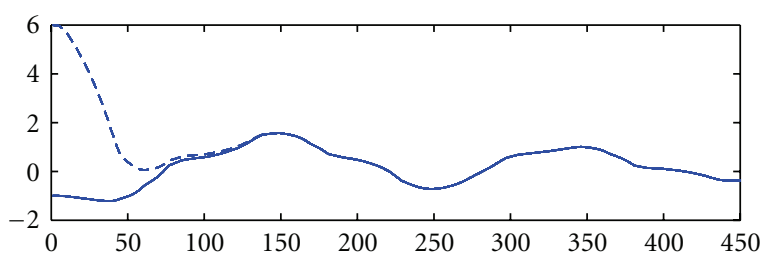

(b)

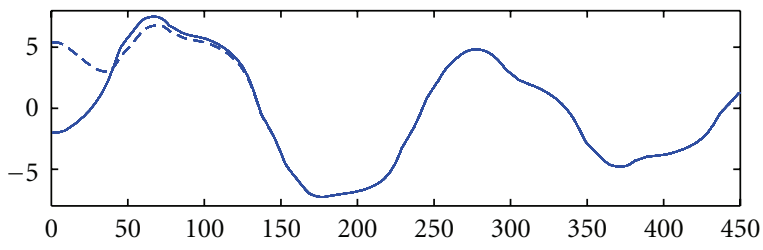

(c)

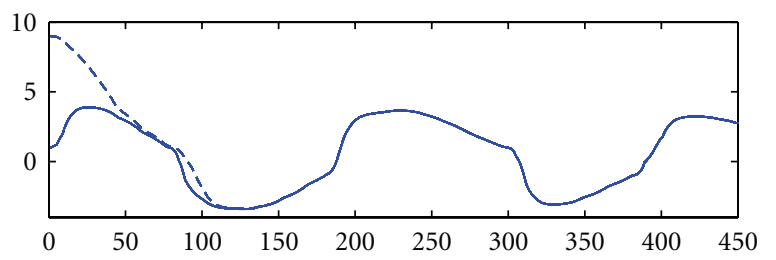

(d)

FIGURE 4: (a) Synchronization of $x_{1}(t)$ and $y_{1}(t)$. (b) Synchronization of $x_{2}(t)$ and $y_{2}(t)$. (c) Synchronization of $x_{3}(t)$ and $y_{3}(t)$. (d) Synchronization of $x_{4}(t)$ and $y_{4}(t)$.

\section{Acknowledgment}

This work is supported by Research Fund Project of the Heze University under Grant: XY10KZ01.

\section{References}

[1] W. Deng, J. Lü, and C. Li, "Stability of N-dimensional linear systems with multiple delays and application to synchronization," Journal of Systems Science and Complexity, vol. 19, no. 2, pp. 149-156, 2006.

[2] E. M. Elabbasy, H. N. Agiza, and M. M. El-Dessoky, "Global synchronization criterion and adaptive synchronization for new chaotic system," Chaos, Solitons and Fractals, vol. 23, no. 4, pp. 1299-1309, 2005.

[3] Q. Zhang, J. Zhou, and G. Zhang, "Stability concerning partial variables for a class of time-varying systems and its applications in chaos synchronization," in Proceedings of the 24th Chinese Control Conference, pp. 135-139, South China University of Technology Press, 2005.

[4] Q. Zhang and G. Jia, "Chaos synchronization of Morse oscillator via backstepping design," Annals of Differential Equations, vol. 22, no. 3, pp. 456-460, 2006.
[5] Q. L. Zhang, "Synchronization of multi-chaotic systems via ring impulsive control," Control Theory and Applications, vol. 27, no. 2, pp. 226-232, 2010.

[6] J. Cao, Z. Wang, and Y. Sun, "Synchronization in an array of linearly stochastically coupled networks with time delays," Physica A, vol. 385, no. 2, pp. 718-728, 2007.

[7] J. Cao and L. Li, "Cluster synchronization in an array of hybrid coupled neural networks with delay," Neural Networks, vol. 22, no. 4, pp. 335-342, 2009.

[8] L. Li and J. Cao, "Cluster synchronization in an array of coupled stochastic delayed neural networks via pinning control," Neurocomputing, vol. 74, no. 5, pp. 846-856, 2011.

[9] H. R. Karimi and P. Maass, "Delay-range-dependent exponential $\mathrm{H} \infty$ synchronization of a class of delayed neural networks," Chaos, Solitons and Fractals, vol. 41, no. 3, pp. 1125-1135, 2009.

[10] J. Cao, D. W. C. Ho, and Y. Yang, "Projective synchronization of a class of delayed chaotic systems via impulsive control," Physics Letters A, vol. 373, no. 35, pp. 3128-3133, 2009.

[11] J. G. Peng and Z. B. Xu, "On asymptotic behaviours of nonlinear semigroup of Lipschitz operators with applications," Acta Mathematica Sinica. Chinese Series, vol. 45, no. 6, pp. 10991106, 2002.

[12] Q. L. Zhang, "Generalized Dahlquist constant with application in impulsive synchronization analysis," in the International Conference on Logistics Systems and Intelligent Management (ICLSIM '10), pp. 1896-1900, January 2010.

[13] G. Jia and Q. Zhang, "Impulsive synchronization of hyperchaotic Chen system," in Proceeding of the 20th Chinese Control and Decision Conference, pp. 123-127, 2008.

[14] J. Huang, C. Li, and Q. Han, "Stabilization of delayed chaotic neural networks by periodically intermittent control," Circuits, Systems, and Signal Processing, vol. 28, no. 4, pp. 567-579, 2009.

[15] J. Yu, H. Jiang, and Z. Teng, "Synchronization of nonlinear systems with delay via periodically intermittent control," Journal of Xinjiang University, vol. 28, no. 3, pp. 310-315, 2010.

[16] Z. Dong, Y. Wang, M. Bai, and Z. Zuo, "Exponential synchronization of uncertain master-slave Lur'e systems via intermittent control," Journal of Dynamics and Control, vol. 7, no. 4, pp. 328-333, 2009.

[17] W. Xia and J. Cao, "Pinning synchronization of delayed dynamical networks via periodically intermittent control," Chaos, vol. 19, no. 1, Article ID 013120, 2009.

[18] J. Huang, C. Li, and Q. Han, "Stabilization of delayed chaotic neural networks by periodically intermittent control," Circuits, Systems, and Signal Processing, vol. 28, no. 4, pp. 567-579, 2009.

[19] B. Shi, D. Zhang, and M. Gai, Theory and Applications of Differential Equations, National Defense Industry Press, 2005.

[20] J. Kuang, Applied Inequalities, Shandong Science and Technology Press, 2004.

[21] L. Xiang, J. Zhou, Z. R. Liu, and S. Sun, "On the asymptotic behavior of Hopfield neural network with periodic inputs," Applied Mathematics and Mechanics, vol. 23, no. 12, pp. 12201226, 2002.

[22] K. Gopalsamy and X. Z. He, "Stability in asymmetric Hopfield nets with transmission delays," Physica D, vol. 76, no. 4, pp. 344-358, 1994.

[23] H. Lu, "Chaotic attractors in delayed neural networks," Physics Letters A, vol. 298, no. 2-3, pp. 109-116, 2002.

[24] M. Gilli, "Strange attractors in delayed cellular neural networks," IEEE Transactions on Circuits and Systems I, vol. 40, no. 11, pp. 849-853, 1993. 
[25] Z.-S. Wang, H.-G. Zhang, and Z.-L. Wang, "Global synchronization of a class of chaotic neural networks," Acta Physica Sinica, vol. 55, no. 6, pp. 2687-2693, 2006.

[26] P. K. Das II, W. C. Schieve, and Z. Zeng, "Chaos in an effective four-neuron neural network," Physics Letters A, vol. 161, no. 1, pp. 60-66, 1991.

[27] C.-J. Cheng, T.-L. Liao, J.-J. Yan, and C.-C. Hwang, "Synchronization of neural networks by decentralized feedback control," Physics Letters A, vol. 338, no. 1, pp. 28-35, 2005.

[28] Z. Q. Wu, F. X. Tan, and S. X. Wang, "Synchronization of the hyper-chaotic system of cellular neural network based on passivity," Acta Physica Sinica, vol. 55, no. 4, pp. 1651-1658, 2006. 

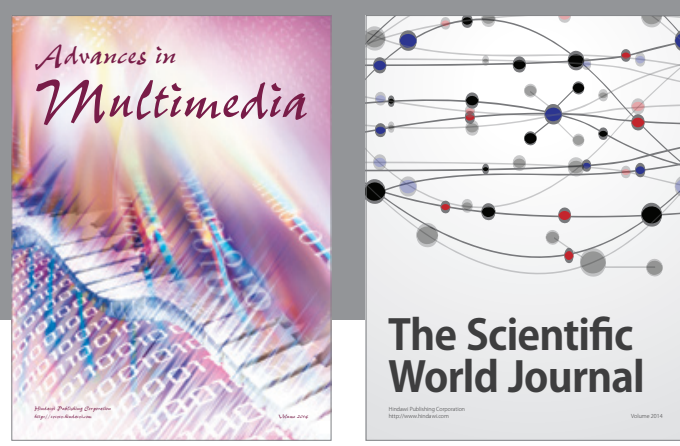

The Scientific World Journal
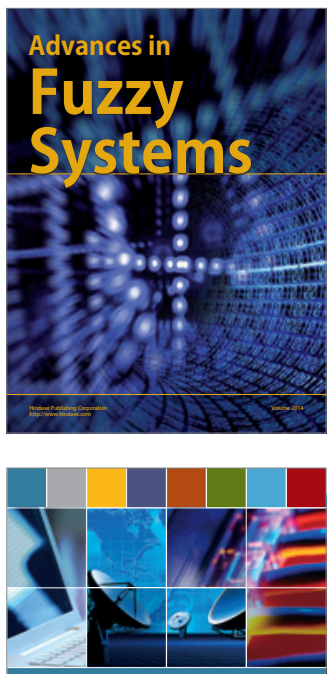

Computer Networks and Communications
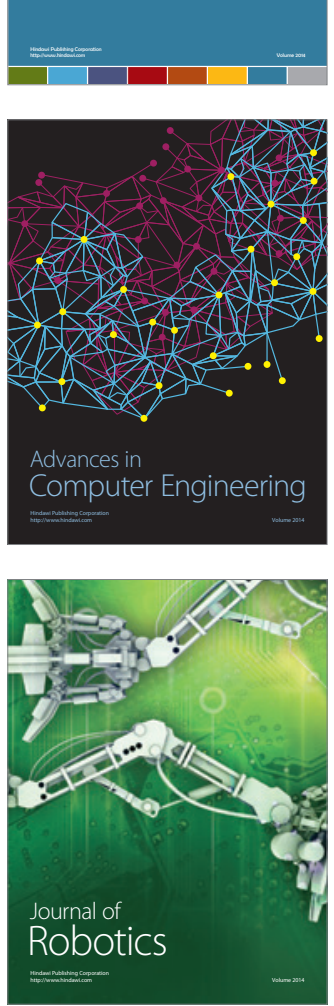
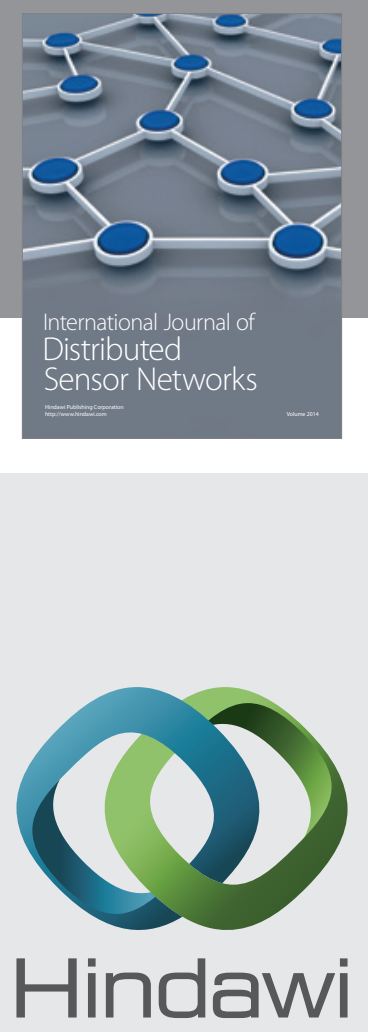

Submit your manuscripts at

http://www.hindawi.com
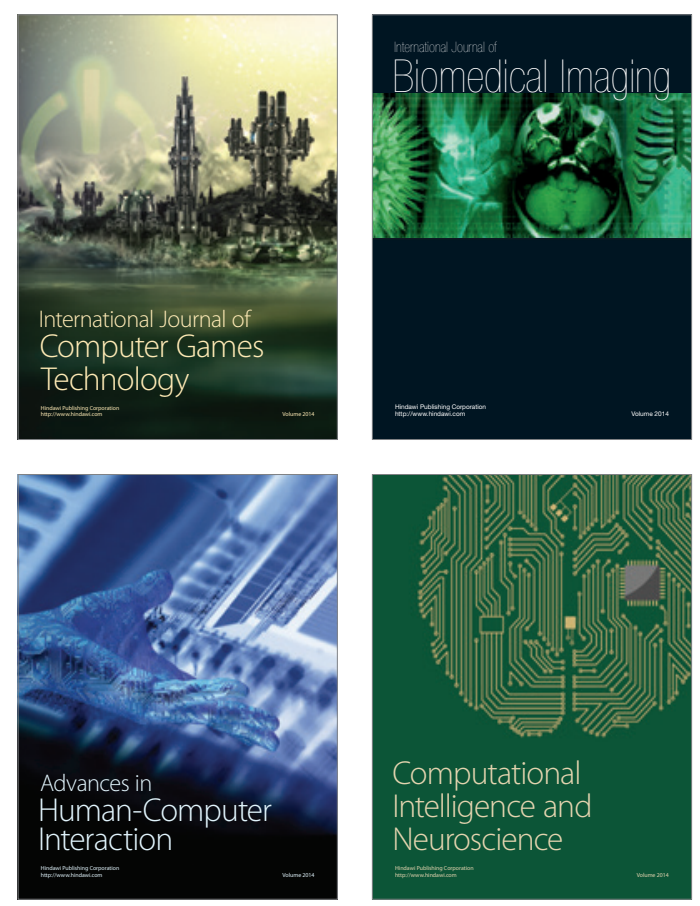
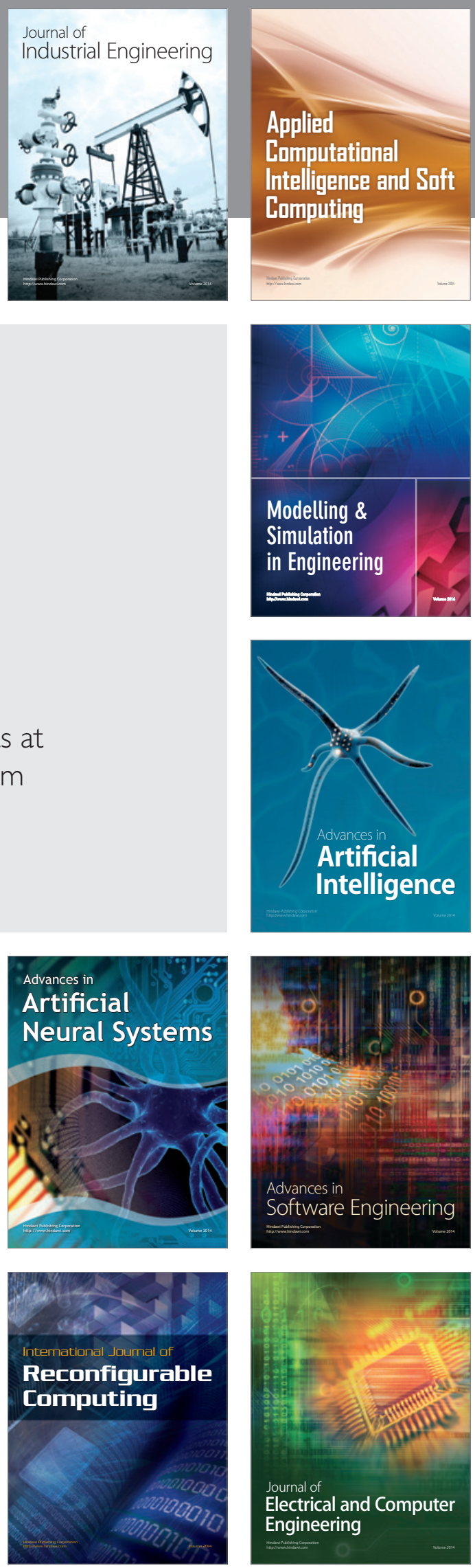